\title{
Correlations of Body Mass Index, Japan-Defined and IDF-Defined Waist Circumference, Visceral and Subcutaneous Fat Area with Metabolic Parameters in Japanese Patients with Type 2 Diabetes
}

\author{
Hidekatsu Yanai ${ }^{*}$, Yuji Hirowatari² \\ ${ }^{1}$ Department of Internal Medicine and Clinical Research Center, National Center for Global Health and \\ Medicine Kohnodai Hospital, Chiba, Japan \\ ${ }^{2}$ Laboratory Sciences, Department of Health Sciences, School of Health and Social Service, Saitama \\ Prefectural University, Saitama, Japan \\ Email: *dyanai@hospk.ncgm.go.jp
}

Received 9 October 2015; accepted 3 November 2015; published 6 November 2015

Copyright ( 2015 by authors and Scientific Research Publishing Inc.

This work is licensed under the Creative Commons Attribution International License (CC BY).

http://creativecommons.org/licenses/by/4.0/

(c) (i) Open Access

\section{Abstract}

Obesity is included in the definition of metabolic syndrome. However, there are many controversies surrounding the evaluation of obesity such as body mass index (BMI) and waist circumference (WC) in the definition of metabolic syndrome among various populations. To understand precisely how various anthropometric indices of obesity influence metabolic parameters, we studied the correlations of BMI, WC (Japanese criteria), WC (IDF criteria), visceral fat area (VFA), subcutaneous fat area (SFA) and the VFA/SFA ratio with metabolic parameters in patients with type 2 diabetes. The influences of BMI and WC on diastolic blood pressure and HDL-cholesterol were larger than VFA, whereas the influences of visceral fat area on triglyceride, very low-density lipoprotein-cholesterol, C-peptide and high-sensitivity C-reactive protein were larger than BMI and WC. WC (IDF) was the strongest determinant of adiponectin among anthropometric indices. Present study showed significant different associations of BMI, Japan- and IDF-defined WC, VFA, SFA and the VFA/SFA ratio with blood pressure, glucose and lipid metabolism, and adipocytokines in Japanese patients with type 2 diabetes.

${ }^{*}$ Corresponding author.

How to cite this paper: Yanai, H. and Hirowatari, Y. (2015) Correlations of Body Mass Index, Japan-Defined and IDF-Defined Waist Circumference, Visceral and Subcutaneous Fat Area with Metabolic Parameters in Japanese Patients with Type 2 Diabetes. Journal of Diabetes Mellitus, 5, 290-294. http://dx.doi.org/10.4236/jdm.2015.54036 


\section{Keywords}

\section{Body Mass Index, Type 2 Diabetes, Visceral Fat Area, Waist Circumference}

\section{Introduction}

Obesity, which is included in the definition of metabolic syndrome, has been considered to be a major risk factor for atherosclerosis [1]. However, there are many controversies surrounding the evaluation of obesity such as body mass index (BMI) and waist circumference (WC) in the definition of metabolic syndrome among various populations [2]. World Health Organization used BMI to evaluate obesity [3]. WC measured at the subjects' umbilical lines has been incorporated into the Japanese criteria for metabolic syndrome [4], whereas WC measured midway between the inferior margin of the ribs and superior border of the iliac crest is performed in the current International Diabetes Federation (IDF) criteria [5]. Recently, we studied the association of BMI and visceral fat area (VFA) with metabolic parameters in Japanese patients with type 2 diabetes, and found significant different associations of BMI and VFA with metabolic parameters [6]. To understand precisely how various anthropometric indices of obesity influenced metabolic parameters, we studied the correlations of BMI, WC (Japanese criteria), WC (IDF criteria), VFA, subcutaneous fat area (SFA) and the VFA/SFA ratio with blood pressure, glucose and lipid metabolism, adipocytokines, albuminuria in patients with type 2 diabetes.

\section{Materials and Methods}

\subsection{Study Participants}

This study was conducted based on the Helsinki Declaration and approved by the Medical Ethics Committee of the National Center for Global Health and Medicine, reference number (NCGM-G-000889). The study participants were patients with type 2 diabetes ( 27 men and 20 women). The mean \pm SD of age, duration of diabetes, height, weight, BMI, systolic and diastolic blood pressure (BP), fasting plasma glucose (FPG), HbAlc, lowdensity lipoprotein-cholesterol (LDL-C), high-density lipoprotein (HDL)-C and triglyceride (TG) of participants were $58.7 \pm 14.1$ years, $9.1 \pm 9.9$ years, $161.2 \pm 7.9 \mathrm{~cm}, 66.1 \pm 9.9 \mathrm{~kg}, 25.4 \pm 3.6 \mathrm{~kg} / \mathrm{m}^{2}, 130.2 \pm 18.1 \mathrm{mmHg}$ and $76.0 \pm 9.3 \mathrm{mmHg}, 157.4 \pm 51.6 \mathrm{mg} / \mathrm{dl}, 7.0 \% \pm 1.4 \%, 100.9 \pm 26.1 \mathrm{mg} / \mathrm{dl}, 51.8 \pm 11.7 \mathrm{mg} / \mathrm{dl}$ and $87.5 \pm 30.3$ $\mathrm{mg} / \mathrm{dl}$, respectively.

\subsection{Anthropometric Measurements}

Height and weight were measured with a rigid stadiometer and calibrated scale (seca 764, seca Co., Ltd, Birmingham, United Kingdom). BMI was calculated as body weight $(\mathrm{kg})$ divided by the square of height $(\mathrm{m})$. WC was measured in a standing posture at the umbilical level (Japanese criteria) and midway between the inferior margin of the ribs and superior border of the iliac crest (IDF criteria) while breathing out.

\subsection{Measurements of VFA and SFA}

We determined VFA and SFA by using the abdominal computed tomography.

\subsection{Blood and Urine Examination}

Venous blood samples were taken after a 12-h overnight fast. Fasting plasma glucose (FPG) was measured using an enzymatic method. Glycated hemoglobin (HbAlc) was measured by high-performance liquid chromatography (HPLC). TG and HDL-C were measured enzymatically using commercially available kits. LDL-C was obtained by the Friedwald formula [7]. Serum C-peptide and high-molecular weight adiponectin were measured by the chemiluminescent immunoassay. Serum high-sensitivity C-reactive protein (hs-CRP) was measured by the enzyme-linked immunosorbent assay. We measured lipoprotein fractions by our developed anion-exchange HPLC [8]. Urine albumin to creatinine ratio (UACR) was measured by the immunonephelometry. 


\subsection{Statistical Analysis}

All statistical analyses were performed using SPSS version 19 (IBM Co., Ltd, Chicago, IL). We analyzed the correlation between anthropometric indices and parameters by the Spearman rank correlation. A $P$ value of $<0.05$ was considered statistically significant.

\section{Results}

\subsection{Correlation between WC and VFA}

WC (Japanese criteria) $(\mathrm{r}=0.914, P<0.001)$ was more significantly correlated with VFA than WC (IDF criteria) $(\mathrm{r}=0.739, P<0.001)$. The differences in the height which WC was measured between Japanese and IDF criteria were significantly correlated with VFA $(r=0.654, P<0.001)$.

\subsection{Correlations between Anthropometric Indices and Parameters}

Any anthropometric indices were not significantly correlated with systolic BP. BMI, WC (Japanese and IDF criteria) were significantly and positively correlated with diastolic BP, however, VFA, SFA and the VFA/SFA ratio were not correlated with diastolic BP (Table 1).

FPG, HbA1c, LDL-C, non-HDL-C were not correlated with any anthropometric indices. HDL-C was significantly and negatively correlated with all measurements of obesity except for the VFA/SFA ratio, the absolute value of correlation coefficient between BMI and HDL-C was the highest. Serum fasting TG, C-peptide and hsCRP were significantly and positively correlated with all measurements of obesity except for the VFA/SFA ratio, the correlation coefficients between VFA and TG, C-peptide and hs-CRP were the highest among measurements of obesity. Serum high molecular weight adiponectin was also significantly and negatively correlated with all measurements of obesity except for the VFA/SFA ratio, the absolute value of correlation coefficient between WC (IDF) and adiponectin was the highest. Urine albumin-to-creatinine ratio was not correlated with any measurements of obesity. The VFA/SFA ratio was not correlated with any metabolic parameters.

HDL-C measured by the HPLC (HPLC-HDL) was significantly and negatively correlated with all measurements of obesity except for the VFA/SFA ratio, the absolute value of correlation coefficient between BMI and HDL-C was the highest (Table 2).

Table 1. Correlations of body mass index (BMI), waist circumference (WC), visceral fat area (VFA), subcutaneous fat area (SFA) and VFA/SFA with metabolic parameters in patients with type 2 diabetes. $r$ indicates correlation coefficient by Spearman rank correlation, and bold values indicate the values showing a statistical significance.

\begin{tabular}{|c|c|c|c|c|c|c|c|c|c|c|c|c|}
\hline & \multicolumn{2}{|c|}{ BMI } & \multicolumn{2}{|c|}{ WC (Japan) } & \multicolumn{2}{|c|}{ WC (IDF) } & \multicolumn{2}{|c|}{ VFA } & \multicolumn{2}{|c|}{ SFA } & \multicolumn{2}{|c|}{ VFA/SFA } \\
\hline & $\mathrm{r}$ & $P$ value & $\mathrm{r}$ & $P$ value & $\mathrm{r}$ & $P$ value & $\mathrm{r}$ & $P$ value & $\mathrm{r}$ & $P$ value & $\mathrm{r}$ & $P$ value \\
\hline systolic BP & -0.006 & 0.966 & -0.002 & 0.987 & 0.022 & 0.885 & -0.039 & 0.792 & -0.1 & 0.502 & 0.012 & 0.938 \\
\hline diastolic BP & 0.317 & 0.03 & 0.301 & 0.04 & 0.415 & 0.004 & 0.182 & 0.22 & 0.211 & 0.154 & -0.182 & 0.22 \\
\hline FPG & 0.003 & 0.985 & -0.068 & 0.65 & 0.033 & 0.827 & -0.118 & 0.431 & -0.116 & 0.438 & -0.008 & 0.957 \\
\hline HbAlc & 0.231 & 0.119 & 0.209 & 0.158 & 0.221 & 0.136 & 0.162 & 0.278 & 0.112 & 0.452 & 0.08 & 0.593 \\
\hline LDL-C & 0.033 & 0.827 & -0.35 & 0.816 & 0.044 & 0.768 & 0.03 & 0.843 & -0.124 & 0.405 & 0.163 & 0.274 \\
\hline HDL-C & -0.474 & 0.001 & -0.464 & 0.001 & -0.459 & 0.001 & -0.33 & 0.023 & -0.352 & 0.015 & 0.151 & 0.31 \\
\hline TG & 0.378 & 0.009 & 0.382 & 0.008 & 0.393 & 0.006 & 0.424 & 0.003 & 0.406 & 0.005 & 0.051 & 0.733 \\
\hline non-HDL-C & 0.132 & 0.377 & 0.07 & 0.641 & 0.147 & 0.325 & 0.136 & 0.361 & -0.009 & 0.95 & 0.16 & 0.282 \\
\hline C-peptide & 0.575 & $<0.001$ & 0.548 & $<0.001$ & 0.591 & $<0.001$ & 0.634 & $<0.001$ & 0.556 & $<0.001$ & 0.013 & 0.931 \\
\hline adiponectin & -0.395 & 0.006 & -0.35 & 0.16 & -0.452 & 0.001 & -0.38 & 0.009 & -0.338 & 0.02 & 0.011 & 0.943 \\
\hline hs-CRP & 0.493 & $<0.001$ & 0.486 & 0.001 & 0.5 & $<0.001$ & 0.575 & $<0.001$ & 0.344 & 0.018 & 0.178 & 0.231 \\
\hline UACR & -0.052 & 0.732 & -0.048 & 0.75 & -0.077 & 0.612 & -0.124 & 0.411 & -0.213 & 0.155 & 0.164 & 0.277 \\
\hline
\end{tabular}


Table 2. Correlations of body mass index (BMI), waist circumference (WC), visceral fat area (VFA), subcutaneous fat area (SFA) and VFA/SFA with each lipoprotein cholesterol and small dense (sd)- and oxidized (ox)-LDL in patients with type 2 diabetes. $r$ indicates correlation coefficient by Spearman rank correlation, and bold values indicate the values showing a statistical significance.

\begin{tabular}{|c|c|c|c|c|c|c|c|c|c|c|c|c|}
\hline & \multicolumn{2}{|c|}{ BMI } & \multicolumn{2}{|c|}{ WC (Japan) } & \multicolumn{2}{|c|}{ WC (IDF) } & \multicolumn{2}{|c|}{ VFA } & \multicolumn{2}{|c|}{ SFA } & \multicolumn{2}{|c|}{ VFA/SFA } \\
\hline & $\mathrm{r}$ & $P$ value & $\mathrm{r}$ & $P$ value & $\mathrm{r}$ & $P$ value & $\mathrm{r}$ & $P$ value & $\mathrm{r}$ & $P$ value & $\mathrm{r}$ & $P$ value \\
\hline HPLC-HDL & -0.564 & $<0.001$ & -0.548 & $<0.001$ & -0.54 & $<0.001$ & -0.45 & 0.002 & -0.44 & 0.002 & 0.106 & 0.479 \\
\hline HPLC-LDL & 0.067 & 0.657 & 0.01 & 0.994 & 0.083 & 0.58 & 0.051 & 0.731 & -0.084 & 0.573 & 0.159 & 0.287 \\
\hline HPLC-IDL & 0.28 & 0.056 & 0.252 & 0.087 & 0.238 & 0.108 & 0.16 & 0.284 & 0.177 & 0.233 & -0.048 & 0.748 \\
\hline HPLC-VLDL & 0.277 & 0.06 & 0.26 & 0.077 & 0.286 & 0.052 & 0.31 & 0.034 & 0.263 & 0.075 & 0.089 & 0.553 \\
\hline sd-LDL & 0.183 & 0.217 & 0.138 & 0.356 & 0.229 & 0.121 & 0.268 & 0.068 & 0.075 & 0.618 & 0.156 & 0.294 \\
\hline ox-LDL & -0.035 & 0.817 & -0.105 & 0.484 & -0.005 & 0.975 & -0.135 & 0.367 & -0.168 & 0.259 & -0.014 & 0.923 \\
\hline
\end{tabular}

Very low-density lipoprotein (VLDL-C) measured by the HPLC was significantly and positively correlated with only VFA. HPLC-LDL-C, HPLC-IDL-C, and atherogenic LDL such as small dense LDL and oxidized LDL were not correlated with any anthropometric indices.

\section{Discussion}

In the metabolic syndrome, the measurement of WC is considered to evaluate visceral obesity. WC (Japanese criteria) was more significantly correlated with VFA than WC (IDF criteria), supporting the validity of Japandefined WC to grasp visceral obesity in Japanese people.

Present study showed that the influence of BMI and WC on diastolic BP, HDL-C was larger than VFA, whereas the influence of VFA on triglyceride, VLDL-C, C-peptide and hs-CRP was larger than BMI and WC. WC (IDF) was the strongest determinant of adiponectin among anthropometric indices. This indicates significant different associations of BMI, WC, and VFA with metabolic parameters and adipocytokines in Japanese patients with type 2 diabetes.

Recently, we reported that the associations of VFA and the VFA/SFA ratio to metabolic parameters in obese Japanese subjects with pre-diabetes [9]. The participants were patients without overt diabetes (12 men and 17 women). The mean \pm SD of age, BMI, FPG and HbAlc were $49 \pm 14$ years, $33.0 \pm 5.6 \mathrm{~kg} / \mathrm{m}^{2}, 101 \pm 16 \mathrm{mg} / \mathrm{dl}$ and $6.2 \% \pm 0.7 \%$, respectively. Although VFA was significantly and positively correlated with systolic BP, VFA was not correlated with any parameters for lipid and glucose metabolism. The VFA/SFA ratio was significantly and positively correlated with serum fasting TG levels. However, the VFA/SFA ratio was not correlated with any metabolic parameters in present study. This suggests a difference in influences of anthropometric indices on metabolic parameters between pre-diabetes and type 2 diabetes.

We have to mention the limitation of our study. The number of subjects was small. To elucidate our hypothesis, further studies, preferably with larger numbers of subjects, will be needed.

\section{Conclusion}

Our study showed significant different associations of BMI, Japan- and IDF-defined WC, VFA, SFA and the VFA/SFA ratio with blood pressure, glucose and lipid metabolism, and adipocytokines in Japanese patients with type 2 diabetes.

\section{Acknowledgements}

This work was supported by a grant from the National Center for Global Health and Medicine (26-112).

\section{References}

[1] Marinou, K., Tousoulis, D., Antonopoulos, A.S., Stefanadi, E. and Stefanadis, C. (2010) Obesity and Cardiovascular Disease: From Pathophysiology to Risk Stratification. International Journal of Cardiology, 138, 3-8. 
http://dx.doi.org/10.1016/j.ijcard.2009.03.135

[2] Matsha, T.E., Hassan, M.S., Hon, G.M., Soita, D.J., Kengne, A.P. and Erasmus, R.T. (2013) Derivation and Validation of a Waist Circumference Optimal Cutoff for Diagnosing Metabolic Syndrome in a South African Mixed Ancestry Population. International Journal of Cardiology, 168, 2954-2955. http://dx.doi.org/10.1016/j.ijcard.2013.03.150

[3] World Health Organization (1999) Definition, Diagnosis and Classification of Diabetes Mellitus and Its Complications: Report of a WHO Consultation. Part 1: Diagnosis and Classification of Diabetes Mellitus. World Health Organization, Geneva.

[4] Examination Committee of Criteria for the Metabolic Syndrome in Japan (2005) Definition and the Diagnostic Standard for Metabolic Syndrome-Committee to Evaluate Diagnostic Standards for Metabolic Syndrome. Nihon Naika Gakkai Zasshi, 94, 794-809. http://dx.doi.org/10.2169/naika.94.794

[5] Alberti, K.G., Zimmet, P. and Shaw, J. (2006) Metabolic Syndrome-A New World-Wide Definition. A Consensus Statement from the International Diabetes Federation. Diabetes \& Metabolism, 23, 469-480. http://dx.doi.org/10.1111/j.1464-5491.2006.01858.x

[6] Yanai, H. and Hirowatari, Y. (2015) Different Associations of Body Mass Index and Visceral Fat Area with Metabolic Parameters and Adipokines in Japanese Patients with Type 2 Diabetes. Diabetes \& Metabolism, 41, 261-262. http://dx.doi.org/10.1016/j.diabet.2015.03.008

[7] Friedewald, W.T., Levy, R.I. and Fredrickson, D.S. (1972) Estimation of the Concentration of Low-Density Lipoprotein Cholesterol in Plasma, without Use of the Preparative Ultracentrifuge. Clinical Chemistry, 18, 499-502.

[8] Hirowatari, Y., Yoshida, H., Kurosawa, H., Doumitu, K. and Tada, N. (2003) Measurement of Cholesterol of Major Serum Lipoprotein Classes by Anion-Exchange HPLC with Perchlorate Ion-Containing Eluent. Journal of Lipid Research, 44, 1404-1412. http://dx.doi.org/10.1194/jlr.D300003-JLR200

[9] Katsuyama, H., Kawaguchi, A. and Yanai, H. (2015) Not Visceral Fat Area But the Ratio of Visceral to Subcutaneous Fat Area Is Significantly Correlated with the Marker for Atherosclerosis in Obese Subjects. International Journal of Cardiology, 179, 112-113. http://dx.doi.org/10.1016/j.ijcard.2014.10.112 